\title{
Inferior Hypogastric Plexus Blockade versus Acupuncture for the Management of Idiopathic Chronic Pelvic Pain: A Randomized Clinical Trial
}

\author{
Magdy M. Amin ${ }^{1}$, Abdou S. Ait-Allah ${ }^{1}$, Ahmed El-Said A. Ali ${ }^{2}$, Rafat A. Salem², Salah Roshdy Ahmed ${ }^{1}$, \\ Mohamed Akhatim Alsammani ${ }^{3,4}$
}

Background: To compare the clinical efficacies of inferior hypogastric plexus blockade and acupuncture in the management of idiopathic chronic pelvic pain (CPP).

Methods: The study included 117 patients with CPP. Group 1 included 62 patients who underwent inferior hypogastric plexus blockade and group 2 included 55 patients who underwent acupuncture. Pain level was assessed using a visual analogue scale (VAS) immediately and at 2, 6, and 12 weeks after treatment.

Results: $\quad$ The preprocedure VAS score was $7.6 \pm 0.15$ in group 1 and $7.7 \pm 0.24$ in group $2(p>0.05)$. Pelvic pain decreased significantly in both groups after treatment, with pretreatment and posttreatment scores of $7.6 \pm 0.15$ and $2.2 \pm 0.88$, respectively, in group $1(p<0.0001)$ and $7.7 \pm 0.24$ and $4.7 \pm 0.11$, respectively, in group $2(p<0.0001)$. However, the decrease in pain scores throughout the clinical follow-up was significantly more in group 1 than in group $2(p<0.0001)$. Complete disappearance of symptoms was achieved in $72.6 \%$ of

At a Glance Commentary

Scientific background of the subject

Pelvic pain is a common gynecological complaint among females of reproductive age. There are many modalities treatment of chronic pelvic pain (CPP) in women. The choice of one of these treatments depends on doctor's preferences, familiarity, and his/her own training, most probably due to lack of clinical trials in this area.

\section{What this study adds to the field}

The study has yielded more evidence that inferior hypogastric plexus blockade provides a better effect in reducing pain intensity and duration of treatment in women with CCP compared to acupuncture. patients in group 1 compared to $54.5 \%$ of patients in group $2(p=0.3737)$. Patients who did not benefit from the treatment were significantly more in group 2 than in group $1(25.5 \%$ vs. $6.5 \%, p=0.0294)$. No complications were reported in both groups.

Conclusion: The study results showed that inferior hypogastric blockade had a $72.6 \%$ success rate and showed a significantly higher effect on reducing pain intensity in a short period of time in the management of CPP, compared to acupuncture.

(Biomed J 2015;38:317-322)

Key words: acupuncture, chronic pelvic pain, complications, efficacy, inferior hypogastric plexus block

$\mathrm{P}$ elvic pain is a common gynecological complaint among females of reproductive age. The intensity of pain may vary from mildly irritating to incapacitating. ${ }^{[1]}$ Chronic pel- vic pain $(\mathrm{CPP})$ is commonly defined as noncyclic pain of at least 6 months duration, localized to the pelvis or anterior abdominal wall, or at or below the umbilicus and at lower

From the ${ }^{1}$ Departments of Obstetrics and Gynecology, Faculty of Medicine, Sohag University, Sohag, Egypt; ${ }^{2}$ Anesthesiology and Algesiology, Faculty of Medicine, Sohag University, Sohag, Egypt; ${ }^{3}$ Department of Obstetrics and Gynecology, College of Medicine, Qassim University, Buraidah, Saudi Arabia; ${ }^{4}$ Department of Obstetrics and Gynecology, College of Medicine, Bahri University, Khartoum, Sudan Received: Sep. 06, 2013; Accepted: Jan. 15, 2015

Correspondence to: Dr. Mohamed Alkhatim Alsammani, Department of Obstetrics and Gynecology, College of Medicine, Qassim University, Buriadah, KSA. P. O. Box: 665. Buraidah 51452, KSA. Tel: 966-568525808; Fax. 966-16-3801228;

Email: m_sammani@yahoo.com

DOI: $10.4103 / 2319-4170.151034$ 
back and buttocks. ${ }^{[2]} \mathrm{CPP}$ is the second most common gynecological presenting complaint, accounting for $13-20 \%$ of gynecological consultations and up to $25 \%$ of the indications for diagnostic laparoscopy. ${ }^{[3]}$ Many etiological factors can contribute to CPP. More than one etiological factor may be found in one patient, and both organic and non-organic causes may be present. ${ }^{[4]} \mathrm{CPP}$ is a problem requiring careful gynecological evaluation to exclude gastrointestinal, orthopedic, urological, neurological, and psychological involvement. ${ }^{[5]}$ CPP with no specific pathological finding is suggested to be due to unrecognized dysfunction of the nervous system, which is often difficult to treat. Medication and surgical intervention may only provide temporary relief. ${ }^{[4]}$ Nonsteroidal anti-inflammatory drugs are used as initial therapy for the treatment of mild pain. They can also be used in combination with opioids and adjuvant analgesics if the intensity of pain worsens. ${ }^{[6]}$ In most cases, good results from acupuncture are achieved by adjunct treatments such as hypnosis, exercise, and transcutaneous electrical stimulation; biofeedback treatment with intensive psychotherapy has been shown to achieve $71 \%$ pain reduction. ${ }^{[7,8]}$ Visceral pain is transmitted via the sympathetic nervous system, regardless of its etiology. Sympathetic nerve blockade was reported to be an effective method for the treatment of pelvic pain. While successful results have been reported with superior hypogastric plexus blockade through an anterior approach, the technique is associated with injury to structures overlying the plexus, such as the bowel, bladder, and common iliac artery. Studies have revealed that acupuncture clinical trials have many problems, including the use of unscientific research protocols and repeated trials of acupuncture, but still papers related to this procedure are increasingly being published in the literature. ${ }^{[9]}$ Meanwhile, inferior hypogastric plexus blockade was proved to be safe and effective if properly performed, but clinical trials of this procedure are scant. ${ }^{[10]}$ The aim of the present study was to compare the efficacy and safety of inferior hypogastric plexus blockade with those of acupuncture for the treatment of idiopathic CPP.

\section{METHODS}

This study was conducted in the Department of Obstetrics and Gynecology and the Pain Clinic of the Department of Anesthesiology and Algesiology, Sohag University Hospital during the period from February 2008 to January 2012.

The study protocol was approved by the faculty of the ethical committee, and informed written consent was obtained from each participating woman.

Women who met the inclusion criteria for CPP underwent thorough history, general and pelvic examinations, and abdominal and transvaginal ultrasonography. Routine investigations were performed. Laparoscopy was performed to exclude organic lesion. Patients were selected according to the following criteria: Age 20 years; parous women; CPP of 6 months duration or longer; no orthopedic, urological, neurological, or psychological problems; no pelvic or abdominal pathological finding; no previous abdominal or pelvic pathological finding; and no pathological findings on laparoscopic examination. Postmenopausal women and those with infertility were excluded from the study. Our variables of interest were maternal age, parity, occupation, pain characteristics, and visual analogue scale (VAS) scores before and after treatment in both groups. The outcome variable we assessed was pain during and after discontinuation of treatment.

A total of 117 patients met the above-mentioned inclusion criteria. The patients were randomly allocated to two groups as follows: 62 patients were assigned to group 1 (the study group) to undergo inferior hypogastric plexus blockade and the remaining 55 patients were assigned to group 2 to undergo acupuncture treatment [Figure 1].

\section{Description of inferior hypogastric plexus blockade technique}

Inferior hypogastric plexus blockade was performed according to the transsacral approach introduced by Schultz in 2007. ${ }^{[10]}$ The patient was placed in the prone position on the radiographic table. An anteroposterior scout view of the sacrum was obtained, and then the $\mathrm{C}$-arm cephalad was tilted to view the sacral foramina "end-on" as circles or semicircles on each side of the midline. Using fluoroscopy, an entrance point was marked on the skin surface $1-2 \mathrm{~cm}$ lateral to the lateral edge of the $\mathrm{S} 2$ or $\mathrm{S} 3$ sacral foramen on the side to be blocked. S2 was the most commonly selected point for its accessibility. After cleaning the skin, a skin wheal was raised over the entrance site to provide a path for anesthesia infiltration toward the targeted sacral foramen. A 3.5-inch, 25 -gauge bent spinal needle was passed through the anesthetized track and advanced down to the lateral aspect of the dorsal sacral foramen until contact was made with bone. The needle was advanced slowly and incrementally under fluo-

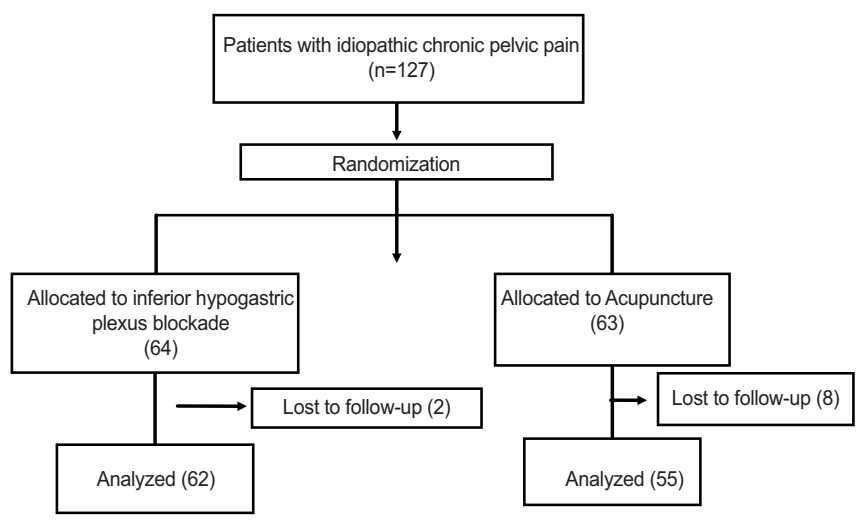

Figure 1: study design flowchart. 
roscopic guidance through the dorsal sacral foramen toward the medial interior edge of the ventral sacral foramen until contact was made with the medial bony edge of the ventral sacral foramen. If sacral paresthesia was encountered, the needle was retracted and rotated slightly to move past the sacral nerve root. Small, incremental doses $(0.1-0.3 \mathrm{ml})$ of $1 \%$ lidocaine during needle advancement improved patient comfort without creating blockade of sacral nerve roots. The needle was maneuvered along the medial edge of the ventral sacral foramen to exit the ventral foramen as medial as possible and anteromedially advanced for another millimeter toward the midline presacral plane. Then, the contrast medium was injected. If the needle is in the optimal position, the contrast should spread cephalad and caudal along the presacral plane conforming to the midline ventral surface of the sacrum. A mixture of $10 \mathrm{ml}$ of $2 \%$ lidocaine and $10 \mathrm{mg}$ of triamcinolone was injected only when proper needle tip position was assured. If the injected contrast medium and medication spread across the midline from the side of the needle placement, then a unilateral block may be adequate. However, contrast spread is more commonly primarily unilateral, necessitating a bilateral needle placement for complete blockade of the right and left inferior hypogastric plexuses.

\section{Description of the acupuncture technique}

An ES-130 Electro-Acupuncture Device (Murakami, Japan) was used for the acupuncture technique. Acupuncture was performed using disposable stainless steel needles $(0.3 \mathrm{~mm}$ diameter, $60 \mathrm{~mm}$ length; Seirin Kasei, Shimizu, Japan) by trained doctors. ${ }^{[8]}$ The acupuncture protocol consisted of a standardized set of acupuncture points applied for $30 \mathrm{~min}$ twice weekly for 6 weeks with electrical stimulation, based on zàng organs stipulated by Traditional Chinese Medicine and meridian theory, as were described by previous researchers ${ }^{[6,11]}$ First, needling was performed in the auricular points, followed by the full-body points. The body acupuncture points selected at each visit included SJ5-wai guan, GB41-zulin qi, LR3-tai chang, LI 4-he gu, SP 8-di ji, and SP 6-san yin jiao. Needling was performed at a depth of 1.5-2.5 inch at each selected point, followed by the full-body points, until a deqi sensation was obtained. ${ }^{[12]}$ Deqi is defined as a feeling of soreness, numbness, distension, or heaviness around the point after the needle was inserted; meanwhile, the practitioner may feel tension around the needle. ${ }^{[13]}$ The needle remained in situ for 25-30 min; during this time, the needle was stimulated with electrical stimulation using low-intensity pulsed currents administered at high frequencies [between 10 and 200 pulses per second (pps)] at the site of pain, and at high intensity and low frequency (lower than 10 pps, usually $2 \mathrm{pps}$ ) in the trigger points. ${ }^{[14]}$ The body acupuncture points selected at each visit included SJ5-wai guan, GB41-zulin qi, LR3-tai chang, LI 4-he gu, SP 8-di ji, and SP 6 -san yin jiao. VAS scores $(1=$ no pain, $2-3=$ mild pain, $4-7=$ moderate pain, $8-10=$ severe disabling pain) were used to assess the severity of pain before the start of treatment and at 2, 4, and 12 weeks after treatment. ${ }^{[14]}$ The pain was also assessed 4 and 12 weeks after discontinuation of treatment in both groups.

\section{Sample size and statistical analysis}

Using an online open epidemiological calculator (http:// www.openepi.com/OE2.3/Menu/OpenEpiMenu.htmcalculator) for sample size estimation for the difference in the mean, ${ }^{[15]}$ the sample sizing assumed the expected difference in the two mean values of VAS after the procedure in the two arms of the study. The expected mean (SD) of the VAS was presumed to be $5.0(0.8)$ and $4.6(0.7)$ following the inferior plexus group and acupuncture procedure, respectively. To achieve $80 \%$ power to detect this difference with a significance level of 5\%, it was estimated that 57 subjects per group would be required. With a withdrawal/non-evaluable subject rate of $10 \%$, a total of 64 subjects per group should be recruited for the required total sample size of 128 subjects.

The Statistical Package for Social Sciences (SPSS 15 for Windows) was used for data recording and statistical analysis. A Chi-square test was used to compare the qualitative data, and the Student $t$-test was used to compare the means of quantitative data. The Fisher's exact test was used when the cell count was less than 5. A $p<0.05$ was considered statistically significant.

\section{RESULTS}

The socio-demographic characteristics of the two groups are shown in Table 1. No significant differences were found between the two groups with regard to their age, parities, occupations, and body mass indices $(p>0.05)$. Both groups had a similar duration of CPP $(10.3 \pm 6.3$ vs. $11.6 \pm 3.4$ months, $p>0.05$ ).

CPP commonly presented as a dull ache $(58.06 \%$ in group 1 vs. $61.8 \%$ in group 2) and involved the whole

Table 1: Comparison of the demographic and biological characteristics of the two groups

\begin{tabular}{lccc}
\hline Characteristics & Group 1 $(n=62)$ & Group 2 $(n=55)$ & $p$ \\
\hline Age in years & $33.82 \pm 7.64$ & $34.42 \pm 3.13$ & $>0.05$ \\
BMI & $26.32 \pm 2.81$ & $25.28 \pm 3.24$ & $>0.05$ \\
Primipara & $10(16.1)$ & $13(23.6)$ & 0.5443 \\
Multipara & $21(33.9)$ & $17(30.9)$ & 0.9547 \\
Grandmultipara & $31(50)$ & $25(45.5)$ & 0.8973 \\
Housewife & $19(30.6)$ & $14(25.5)$ & 0.7880 \\
Employee & $43(69.4)$ & $41(74.5)$ & 0.9132 \\
\hline
\end{tabular}

Data are presented as mean \pm SD and number (percentage).

Abbreviation: BMI: Body mass index 
pelvis (50\% in group 1 and 50.9\% in group 2), with no statistically significant differences between the two groups ( $p=0.9555$ and $p=0.9551$, respectively).

The pain was localized in most of the cases $(69.35 \%$ in group 1 and $61.8 \%$ in group 2) and confined to the lower back in $25.8 \%$ of the subjects in group 1 and $25.4 \%$ of the subjects in group 2. However, no statistically significant difference in pain characteristics was observed between the two groups [Table 2].

The VAS score before intervention was $7.6 \pm 0.15$ in group 1 compared to $7.7 \pm 0.24$ in group 2 , with no statistically significant difference $(p>0.05)$. Although both groups had statistically significant reduction in pain levels throughout the follow-up period, group 1 reported a more significant reduction in pain level when compared to group $2(p<0.05)$ [Table 3].

The results evaluated after 4 and 12 weeks of the interventions are presented in Table 4. After 1 month of treatment, group 1 reported a higher frequency of complete pain relief than that reported by group 2 (61.2\% vs. $40.09 \%)$, although the difference between the groups was not significant $(p=0.2487)$. After 3 months, complete disappearance of symptoms was reported by $72.5 \%$ of the patients in group 1 and $54.5 \%$ of the patients in group $2(p=0.420)$. The number of patients who reported that there was no change in the level of pain after 1 and 3 months of intervention was significantly higher in group 2 than in group 1 ( $9.6 \%$ vs. $30.9 \%, p=0.033$ and $25.5 \%$ vs. $6.5 \%, p=0.0294$, respectively). The number of patients who did not benefit from the treatment was significantly higher in group 2 than in group $1(25.5 \%$ vs. $6.5 \%, p=0.0294)$. No complications were reported in both groups during the intervention and the follow-up period.

\section{DISCUSSION}

CPP is a common complaint among women and is a major public health concern worldwide. ${ }^{[16]}$ It causes frustration among patients, which often has a major impact on quality of life. ${ }^{[13]}$ Despite the high prevalence of CPP, its management remains a major challenge to clinicians mainly because of the lack of understanding of its natural history, etiology, and pathogenesis.

In the present study, we compared the efficacy and safety between inferior hypogastric plexus blockade and acupuncture as a nonconventional therapeutic modality for the management of chronic idiopathic pelvic pain. We found that the pain scores were significantly reduced in both groups. However, a more significant reduction of pain score was achieved by inferior hypogastric plexus blockade than acupuncture $(p<0.0001)$. The post-intervention pain score in the women who underwent inferior hypogastric plexus blockade was $2.2 \pm 0.88$, as compared to $4.7 \pm 0.11$ found among the women in the acupuncture group $(p<0.0001)$,
Table 2: Comparison of pain characteristics between the two groups

\begin{tabular}{lccc}
\hline Characteristics & Group 1 $(n=62)$ & Group 2(n=55) & $p$ \\
\hline $\begin{array}{l}\text { Duration in months } \\
\text { Site }\end{array}$ & $10.3 \pm 6.3$ & $11.6 \pm 3.4$ & $>0.05$ \\
$\quad$ Suprapubic & $13(21)$ & $11(20)$ & 0.9163 \\
$\quad$ One or both iliac fosse & $18(29)$ & $16(29.1)$ & 0.9959 \\
$\quad$ Whole pelvis & $31(50)$ & $28(50.9)$ & 0.9551 \\
Type & & & \\
$\quad$ Stitching & $22(35.5)$ & $19(34.5)$ & 0.9413 \\
Colicky & $4(6.4)$ & $2(3.6)$ & 0.8139 \\
$\quad$ Dull ache & $36(58.1)$ & $34(61.8)$ & 0.9555 \\
Referred & & & \\
$\quad$ Lowerback & $16(25.5)$ & $14(25.5)$ & 0.9733 \\
$\quad$ Lowerlimbs & $3(4.8)$ & $2(3.6)$ & 0.7584 \\
$\quad$ Not referred & $43(69.4)$ & $39(70.9)$ & 0.9388 \\
\hline
\end{tabular}

Data are presented as mean \pm SD and number (percentage)

Table 3: Comparison of visual analogue scale scores before and after treatment between both groups

\begin{tabular}{lccc}
\hline Characteristics & Group 1 $(n=62)$ & Group 2 $(n=55)$ & $p$ \\
\hline Before treatment & $7.6 \pm 0.15$ & $7.7 \pm 0.24$ & NS \\
One hour & $2.2 \pm 0.88$ & $7.8 \pm 0.24$ & 0.001 \\
2 weeks & $2.2 \pm 0.88$ & $6.3 \pm 0.14$ & $<0.05$ \\
6 weeks & $2.2 \pm 0.88$ & $5.2 \pm 0.06$ & $<0.05$ \\
12 weeks & $2.2 \pm 0.88$ & $4.7 \pm 0.11$ & $<0.05$ \\
\hline
\end{tabular}

Data are presented as mean $\pm \mathrm{SD} ; p<0.05$

Table 4: Pain outcome at 1 and 3 months after discontinuation of treatment in the two groups

\begin{tabular}{lccc}
\hline Pain status after 4 weeks & Group 1 $(n=62)$ & Group 2 $(n=55)$ & $p$ \\
\hline Completely relieved & $38(61.3)$ & $22(40)$ & 0.6899 \\
Partially relieved & $14(22.6)$ & $13(23.6)$ & 0.9149 \\
No change & $6(9.7)$ & $17(30.9)$ & 0.0330 \\
No table to decide & $4(6.5)$ & $3(5.5)$ & 1.0000 \\
Pain status after 12 weeks & & & \\
$\quad$ Completely relieved & $45(72.6)$ & $30(54.5)$ & 0.3737 \\
Partially relieved & $11(17.7)$ & $10(18.2)$ & 0.9588 \\
No change & $4(6.5)$ & $14(25.5)$ & 0.0294 \\
No table to decide & $2(3.2)$ & $1(1.8)$ & 1.000 \\
\hline
\end{tabular}

demonstrating a $72.6 \%$ success rate in the inferior hypogastric plexus blockade group (evident by the disappearance of all symptoms) compared to $54.5 \%$ in the acupuncture group ( $p=0.3737)$. Moreover, $25.5 \%$ of the women in the acupuncture group compared to $6.5 \%$ of the women in the inferior hypogastric plexus blockade group $(p=0.0294)$ reported that they did benefit from the treatment, indicating that acupuncture when used for CPP was associated with a high failure rate, although some studies demonstrated a high success rate with acupuncture but not for CPP.

These results indicate that inferior hypogastric plexus blockade was superior to acupuncture in producing fast pain relief and significant reduction of pain intensity throughout the study period, which reflect the patients' satisfaction. The 
high failure rate in the acupuncture group after discontinuation of treatment may be attributed to the fact that CPP is primarily a reflexive pain of nociceptive origin. Therefore, it may respond well to inferior hypogastric plexus blockade.

The inferior hypogastric plexus blockade technique requires familiarity with the sacral anatomy and expertise in fluoroscopy and refined needle technique. The procedure was proved to effective and safe for diagnosis and treatment of CPP ${ }^{[10]}$ By contrast, reports on the safety and efficacy of acupuncture are diverse and inconclusive, and its use is associated with some adverse reactions.

One series that used acupuncture for 1 year in 43 patients with primary dysmenorrhea showed a $91 \%$ improvement in symptoms and $41 \%$ decrease in analgesic use. ${ }^{[17]}$ Furthermore, a meta-analysis performed to determine the effectiveness and safety of acupuncture for pain in endometriosis concluded that evidence to support the effectiveness of acupuncture for pain in endometriosis is limited. ${ }^{[18]}$ This clinical improvement in pain with acupuncture can be enhanced when infrared laser acupuncture is used. ${ }^{[19,20]}$ Compared with acupuncture, hypogastric plexus blockade for pain relief in endometriosis was shown in one study to significantly relieve pain immediately after establishing the block, which is in good agreement with our findings. ${ }^{[21]}$ This high success rate obtained in acupuncture therapy for dysmenorrhea might hold good for the treatment of pain due to conditions other than CPP, as the nature of pain differs according to etiology.

The success rate in our study is similar to that reported by Schultz,${ }^{[10]}$ who introduced the transsacral approach to blockade of the inferior hypogastric plexus. In his series, which involved 11 patients who were treated with inferior hypogastric plexus blockade for $\mathrm{CPP}$, he reported a success rate of $73 \%$, with immediate satisfactory results and no complications. In a case of CPP treated with inferior hypogastric plexus blockade, clinical follow-up showed total pain reduction. ${ }^{[22]}$

Previous studies have shown that inferior hypogastric plexus blockade plays a diagnostic role in the management of chronic pelvic and perineal pains by providing information that serves as guide for more specific global pain management procedures, particularly when the pain is of neoplastic origin. ${ }^{[23,24]}$

The minor differences in the success rate of this technique between different studies may be due to technical variation and operator experience, as the procedure requires knowledge of the pelvic anatomy.

Transsacral approach has a similar success rate, with no complications, as that of anterior superior hypogastric plexus blockade, but the latter is associated with the risks of injury to the overlying nerve, bowel, bladder, and common iliac artery. ${ }^{[10]}$ Thus, transsacral approach for inferior hypogastric plexus blockade should be considered as an alternative procedure for the management of CPP.

\section{Conclusions}

We conclude based on the findings of the study that inferior hypogastric blockade had $72.6 \%$ success rate and a significant effect on pain intensity reduction in a short period in the management of CPP in women, when compared with acupuncture. However, further study is warranted to support this finding.

\section{REFERENCES}

1. Mathias SD, Kuppermann M, Liberman RF, Lipschutz RC, Steege JF. Chronic pelvic pain: Prevalence, health-related qualityof life, and economic correlates. Obstet Gynecol 1996;87:321-7.

2. Issa W, Roumeguere TE, Bossche MV. Chronic pelvic pain syndrome. Rev Med Brux 2013;34:29-37.

3. Lamvu G, Williams R, Zolnoun D, Wechter ME, Shortliffe A, Fulton $\mathrm{G}$, et al. Longterm outcomes after surgical and nonsurgical management of chronic pelvic pain: One year after evaluation in a pelvic pain specialty clinic. Am J Obstet Gynecol 2006;195:591-8

4. Ghaly AF, Chien PW. Chronic pelvic pain: Clinical dilemma or clinician's nightmare. Sex Transm Infect 2000;76:419-25.

5. Forbes P. Diagnosis and treatment of chronic pelvic pain. Practitioner 1998;242:120-3, 125.

6. Lee SW, Liong ML, Yuen KH, Leong WS, Chee C, Cheah PY, et al. Acupuncture versus sham acupuncture for chronic prostatitis/chronic pelvic pain. Am J Med 2008;121:79.e1-7.

7. Hogeboom CJ, Sherman KJ, Cherkin DC. Variation in diagnosis and treatment of chronic low back pain by traditional Chinese medicine acupuncturists. Complement Ther Med 2001;9:154-66.

8. Honjo H, Naya Y, Ukimura O, Kojima M, Miki T. Acupuncture on clinical symptoms and urodynamic measurements in spinal-cord-injured patients with detrusor hyperreflexia. Urol Int 2000;65:190-5.

9. Sluka KA, Walsh D. Transcutaneous electrical nerve stimulation: Basic science mechanisms and clinical effectiveness. J Pain 2003;4:109-21

10. Schultz DM. Inferior hypogastric plexus blockade: A transsacral approach. Pain Physician 2007;10:757-63.

11. Vincent K. Chronic pelvic pain in women. Postgrad Med J 2009;85:24-9.

12. Capili B, Anastasi JK, Geiger JN. Adverse event reporting in acupuncture clinical trials focusing on pain. Clin J Pain 2010;26:43-8.

13. Capodice JL, Jin Z, Bemis DL, Samadi D, Stone BA, Kapan S, et al. A pilot study on acupuncture for lower urinary tract symptoms related to chronic prostatitis/chronic pelvic pain. Chin Med 2000;2:1.

14. Ahn AC, Schnyer R, Conboy L, Laufer MR, Wayne PM. Electrodermal measures of Jing-Well points and their clinical relevance in endometriosis-related chronic pelvic pain. J Altern Complement Med 2009;15:1293-305. 
15. Available from: http://www.openepi.com/OE2.3/menu/openEpiMenu. htm. [Last accessed on 2015 Apr 01].

16. Geng LL, Lin RS, Sun XY, Wu L, Zhong MM, Zhao B, et al. Critical appraisal of randomized clinical trials in Chinese Acupuncture and Moxibustion from 2000 to 2006. Zhongguo Zhen Jiu 2008;28:439-43.

17. Helms JM. Acupuncture for the management of primary dysmenorrhea. Obstet Gynecol 1987;69:51-6.

18. Magalhães J. Acupuncture for pain in endometriosis. Sao Paulo Med J 2013;131:439.

19. Glazov G, Yelland M, Emery J. low-dose laser acupuncture for non-specific chronic low back pain: A double-blind randomised controlled trial. Acupunct Med 2014;32:116-23.

20. McKee MD, Kligler B, Fletcher J, Biryukov F, Casalaina W,
Anderson B, et al. Outcomes of acupuncture for chronic pain in urban primary care. J Am Board Fam Med 2013;26:692-700.

21. Kanazi GE, Perkins FM, Thakur R, Dotson E. New technique for superior hypogastric plexus block. Reg Anesth Pain Med 1999;24:473-6.

22. Choi HS, Kim YH, Han JW, Moon DE. A new technique for inferior hypogastric plexus block: A coccygeal transverse approach -A case report. Korean J Pain 2012;25:38-42.

23. Plancarte R, Amescua C, Patt RB, Aldrete JA. Superior hypogastric plexus block for pelvic cancer pain. Anesthesiology 1990;73:236-9.

24. Rigaud J, Delavierre D, Sibert L, Labat JJ. Sympathetic nerve block in the management of chronic pelvic and perineal pain. Prog Urol 2010;20:1124-31. 